\title{
LOS SOBRECOSTOS LOGÍSTICOS EN LA IMPORTACIÓN DE VEHÍCULOS NUEVOS DE TOYOTA 2013 - 2017
}

\author{
LOGISTIC OVERLOADS ON THE IMPORT OF NEW VEHICLES FROM TOYOTA \\ 2013-2017
}

BASILIA Jessica SAntome Jiménez Universidad Nacional Mayor de San Marcos Lima, Perú

ORCID: $h$ ttp://orcid.org/0000-0003-1462-0086 Correo electrónico: jessysantome@gmail.com

\section{RESUMEN}

Objetivo: Realizar una investigación sobre los principales sobrecostos logísticos que conciernen a la importación de vehículos nuevos de Toyota para minimizar el impacto en dichos procedimientos aduaneros. Método: Se empleó una investigación científica no experimental, debido a que es un tema relacionado a las ciencias sociales, por ese motivo se usaron teorías demostradas como científicas para la sustentación de esta investigación. El tipo de diseño de la investigación es correlacional ya que se examinaron los efectos que tiene una variable sobre la otra. Para lograr este objetivo también se utilizó información proveniente de encuestas de 35 personas que trabajan tanto en la misma empresa Toyota, así como, en la agencia de aduanas que realiza los despachos. Resultados: Se determinan los principales sobrecostos logísticos en el despacho aduanero de importación de vehículos en la empresa Toyota, presentándose estos sobrecostos por conceptos de demoras en un $80 \%$ y en cuanto a riesgos en el terminal portuario representando un $20 \%$ de los sobrecostos. Conclusiones: Será útil para futuros estudios de análisis relacionados a temas de eficiencia, productividad y mejora continua en procedimientos de despachos aduaneros, para estar alineados con las directrices propuestas por los principales organismos internacionales como son la OMA, OMC y la CAN.

Palabras clave: Sistema toyotista; vehículos; importación; Toyota; sobrecostos logísticos.

\begin{abstract}
Objective: To carry out an investigation on the main logistic cost overruns concerning the importation of new Toyota vehicles in order to minimize the impact on customs procedures. Method: A non-experimental scientific research was used since it is a topic related to the social sciences. For that reason, the theories shown as scientific were used for the support of this research. The type of research design is correlational since the effects of one variable on the other were examined. To achieve this objective, information was also used from surveys of 35 people who work both in the same Toyota company, as well as in the customs agency that carries out the dispatches. Results: The main logistic cost overruns are determined at the customs office of import of vehicles in the Toyota company, presenting cost overruns for $80 \%$ delays and at the port terminal in terms of risks representing $20 \%$ of the cost overruns. Conclusions: This work will be useful for future analysis studies related to issues of efficiency, productivity and continuous improvement in customs clearance procedures, to be aligned with the guidelines proposed by the main international organizations such as the World Customs Organization (WCO), the World Trade Organization (WTO) and The Andean Community (CAN).
\end{abstract}

Keywords: Toyota system; vehicles; import; Toyota; logistic cost overruns.

(c) Los autores. Este artículo es publicado por la Revista Quipukamayoc, Universidad Nacional Mayor de San Marcos. Este es un artículo de acceso abierto, distribuido bajo los términos de la Licencia Creative Commons Atribución-NoComercial-Compartirlgual 4.0 Internacional.(http://creativecommons.org/licenses/by-nc-sa/4.0/), que permite el uso no comercial, distribución y reproducción en cualquier medio, siempre que la obra original sea debidamente citadas. 


\section{INTRODUCCIÓN}

En un mundo tan cambiante como en el que se vive en estos momentos, el ser competitivo no es una opción sino una obligación, es la diferencia si existes o no en el mercado y para llevarse a cabo este propósito se deben implementar en las empresas mejoras continuas, gestión eficiente del conocimiento y la adaptación al cambio (García, 2013)

Las empresas necesitan diferenciarse para hacerle frente no solo a su competencia sino también al mundo globalizado, cambiante y altamente competitivo en el que existen, es por ello que se destaca la empresa Toyota, como un caso de éxito, que tiene el prestigio de gozar del liderazgo y éxito de la marca a nivel internacional (Hermosilla, 2015)

La empresa pionera en el tema de eficiencia y mejora continua siempre ha sido la empresa japonesa Toyota que ha sobresalido por emplear técnicas que evitan los cuellos de botellas que se pueden presentar en algunas áreas de la empresa para optimizar tiempos y costos, diferenciándose de esa manera de su competencia (Huamán, 2010)

El sistema creado por Toyota es un aporte de suma importancia para la industria, ya que los beneficios que trae consigo son de gran relevancia para hacer más eficiente a la empresa generando con ello reducciones significativas en los inventarios, stocks y defectos (Pellegrini, 2011)

Por otro lado, el sistema toyotista, es un sistema que tiene dos pilares fundamentales que lo hacen único, el primer pilar es el just in time, que significa entregar a tiempo el vehículo al cliente en el momento adecuado en las condiciones pactadas y el segundo pilar en el que se fundamenta dicho sistema, es el jidoka, palabra japonesa, que significa la eliminación de los defectos y desperdicios (Álvarez, 2012)

El modelo toyotista, también denominado "modelo flexible", es un modelo que si bien es cierto fue creado para producir en grandes cantidades, pero también con una forma diferencia de producción adaptándose con ello a los cambios que podrían suscitarse en la demanda de los productos (Rodríguez, 2013)

Como menciona Loaiza (2007), una mejora en la empresa, permite modificar y simplificar procesos, y esto depende de toda la organización, es decir, todos los miembros de la organización deben estar involucrados en la mejora para de esta manera incrementar la calidad y competitividad de la organización. Los beneficios que devienen de implementar una mejora son la primordialmente la disminución de tiempo, minimización de costos y recursos.
Las empresas de hoy en día han añadido para las descripciones de cargos de responsabilidad logística, elementos esenciales para desempeñarse en esa área como son principalmente, cumplir con metas de reducción de costos de almacenaje y distribución, reducción de errores y logística enfoca al cliente, por mencionar las más importantes, lo cual indica que una empresa para ser competitiva en el mercado actual, debe siempre buscar la eficiencia en sus procesos y una de las formas es la de reducir al mínimo los sobrecostos logísticos básicamente los conceptos de almacenaje y distribución, ya que, ello se ve reflejado en el precio final al cliente o consumidor (Quevedo, 2010).

Las formas de hacerles frente a los sobrecostos logísticos que dan resultados muy positivos son por ejemplo la implementación de nuevos centros de distribución que se manejen de una forma eficiente; la implementación del cross docking que permite una minimización y/o extinción del sobrecosto de almacenaje e inventarios; la tercerización u outsourcing de aquellas operaciones que no son el core del negocio; renegociación de fletes al contratar por volúmenes grandes de carga y con el apoyo de la tecnología que brindan muchas herramientas informáticas de última generación brindando soporte para que la gestión de almacenes sea mucho más eficiente que con otros métodos. (Nickl, 2005)

Un aspecto muy importante de mencionar, es que, si se desean realizar mejoras en bienestar de todo el país, cada uno de los actores participantes deben dar su máximo potencial tanto en el sector privado, así como en el sector público, como lo mencionan Bariandarán, Calderón, Chávez y Coello (2013), en su plan estratégico del sector automotriz en el Perú - vehículos ligeros y comerciales, en la cual destaca la importancia de la inversión en infraestructura del puerto del Callao, lo cual incrementa la competitividad y puede incrementar las posibilidades que este pueda llegar a ser un puerto hub para América Latina comparable con los puertos europeos, también se pueden apreciar las siguientes conclusiones más importantes: que a través de la modernización del sector automotriz se contará con uno de los parques automotores con menor impacto ambiental en la región, mejorando la calidad de vida de la población, con ello se garantiza el desarrollo y crecimiento del país.

Para esto, según lo mencionado por Bloch (2012), los operadores de comercio exterior como principalmente lo son los terminales portuarios y almacenes asumen determinadas responsabilidades cuando las mercancías se encuentran dentro de sus instalaciones ya que ante cualquier siniestro y/o incidente serán estos operadores los responsables para responder asumiendo con ello los riesgos que conlleva. 


\section{MATERIALES Y MÉTODOS}

Según Hernández, Fernández y Baptista (2014), este trabajo es una investigación científica siendo de esa manera No Experimental, por cuanto presenta la teorización integral a través de principios, normas, conceptos, procesos y procedimientos.

El tipo de diseño de la investigación es Correlacional, debido a que se examinó los efectos de las variables, asumiendo que la variable 1 ha ocurrido señalando efectos sobre la variable 2 o teniendo relación con respecto a la otra.

Es de tipo transversal por que la recolección de datos se realizó en un solo momento, en un tiempo único.

Las unidades de análisis fueron las personas que se encuentran en el área de importación de la empresa Toyota y la agencia de aduanas que se encargan de realizar los respectivos despachos aduaneros de importación de la empresa TOYOTA DEL PERU S.A. es decir, entre los años 2013 hasta el 2017.

La población de este trabajo de investigación estuvo conformada por las personas del área solo y exclusivamente de importación de la empresa Toyota Del Perú S.A y las personas que trabajan en la empresa TLI Aduanas S.A.C que es la agencia de aduanas que se encarga de tramitar sus importaciones y realizar el despacho aduanero de importación durante el período 2013-2017. Sumadas estas personas, se llegaron a un total de 35 personas. Al ser una población pequeña, no se seleccionará una muestra representativa, sino que se trabajará con toda la población.
Las técnicas de recolección de datos fueron las encuestas que se les aplicó a la población, entrevistas a profundidad y revisión documental.

\section{RESULTADOS}

En el estudio, luego de realizar las encuestas a las personas encargadas del área de importación de la empresa Toyota, entrevistas y revisión documentaria se determinaron una serie de hallazgos con referencia a los principales sobrecostos logísticos que son los puntos críticos para realizar mejoras continuas en los despachos aduaneros de importación de los vehículos nuevos de dicha empresa.

De acuerdo a la tabla 1, se puede indicar que el coeficiente de correlación de Spearman el cual se utilizó, tiene un rango de variación de entre $-1 \mathrm{a}+1$, representando con ello las diferentes correlaciones que existen entre las variables, las cuales pueden ser positivas o negativas. En este caso, al comparar las dos variables se muestra un valor de 0,108 el cual demuestra que existe una correlación positiva muy baja entre las variables sobrecostos logísticos y tiempo de demora del despacho aduanero de importación de los vehículos nuevos de la empresa Toyota, además de mostrarnos una significancia bilateral de 0,538 aplicándose a una población de 35 sujetos.

En la tabla 2 y figura 1, se muestra que, de acuerdo a las encuestas realizadas a la población de 35 personas, el $80 \%$ de ellas, es decir, 28 personas, indican que efectivamente existe una relación entre las variables sobrecostos logísticos y tiempo de demora en el despacho aduanero de im-

Tabla 1

Correlación de variables Sobrecostos logísticos y Tiempo de despacho

\begin{tabular}{|c|c|c|}
\hline \multicolumn{2}{|c|}{$\begin{array}{l}\text { Los sobrecostos logísticos se correlacionan con el tiempo del despacho aduanero de importación } \\
\text { de los vehículos nuevos de la empresa Toyota }\end{array}$} & \multirow{2}{*}{$\begin{array}{c}\text { Tiempo de despacho aduanero } \\
0,108\end{array}$} \\
\hline \multirow{3}{*}{ Los sobrecostos logísticos } & Coeficiente de correlación & \\
\hline & Sig. (bilateral) & 0,538 \\
\hline & $\mathrm{N}$ & 35 \\
\hline
\end{tabular}

Fuente: Elaboración propia en SPSS a partir de encuestas.

Tabla 2

Los sobrecostos logísticos se correlacionan con el tiempo de demora del despacho aduanero

\begin{tabular}{lcc}
\hline \multicolumn{1}{c}{ Respuestas } & Frecuencia & Porcentaje \\
\hline De acuerdo & 28 & $80 \%$ \\
Indiferente & 5 & $14 \%$ \\
En desacuerdo & 2 & $6 \%$ \\
TOTAL & 35 & $100 \%$ \\
\hline
\end{tabular}

Fuente: Elaboración propia a partir de encuestas. 
portación de los vehículos nuevos que importa la empresa Toyota del Perú S.A.

Luego de analizar las tablas 1 y 2 se puede determinar que la correlación que existe entre las variables mencionadas líneas arriba, es directa, ya que luego de aplicar la correlación de Spearman en el programa estadístico del SPSS, el signo es positivo, lo cual muestra que si una de ellas aumenta la otra también lo hará.

Según lo mostrado en la tabla 3, se puede determinar que el coeficiente de correlación de Spearman el cual se utilizó, muestra un valor de 0,178 el cual demuestra que existe una correlación positiva muy baja entre las variables "sobrecostos logísticos del traslado de los vehículos" y el "tiempo de demora del despacho aduanero" de importación de los vehículos nuevos de la empresa Toyota, además de mostrarnos una significancia bilateral de 0,307 aplicándose a una población de 35 sujetos.

De acuerdo a la tabla 4 y figura 2, los sobrecostos logísticos del traslado de los vehículos se correlacionan con el tiempo de demora del despacho aduanero, ya que, de acuerdo a las respuestas de los encuestados, un $83 \%$ indica que se encuentra de acuerdo con ello, el 11\% se muestra indiferente y solo el $6 \%$ está en desacuerdo.

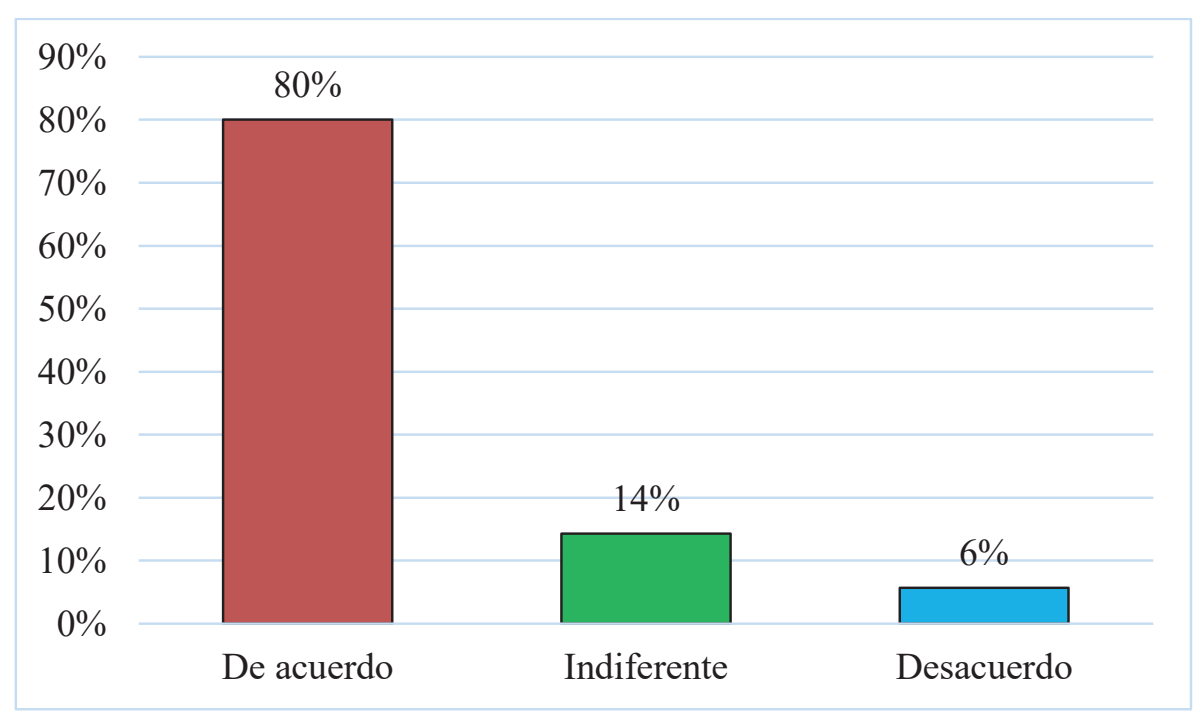

Figura 1. Los sobrecostos logísticos se correlacionan con el tiempo de demora del despacho aduanero

Fuente: Elaboración propia a partir de encuestas.

Tabla 3

Los sobrecostos logísticos del traslado de los vehículos se correlacionan con el tiempo del despacho

\begin{tabular}{lll}
\hline \multicolumn{2}{l}{ Los sobrecostos logísticos del terminal portuario se correlaciona con el tiempo del despacho aduanero } & Tiempo del despacho aduanero \\
\hline \multirow{2}{*}{$\begin{array}{l}\text { Los sobrecostos logísticos del } \\
\text { terminal portuario }\end{array}$} & 0,178 \\
& Sig. (bilateral) & 0,307 \\
\hline
\end{tabular}

Fuente: Elaboración propia en SPSS a partir de encuestas.

Tabla 4

Los sobrecostos logísticos del traslado de los vehículos se correlacionan con el tiempo de demora del despacho aduanero

\begin{tabular}{lcc}
\hline Respuestas & Frecuencia & Porcentaje \\
\hline De acuerdo & 29 & $83 \%$ \\
Indiferente & 4 & $11 \%$ \\
En desacuerdo & 2 & $6 \%$ \\
TOTAL & 35 & $100 \%$ \\
\hline
\end{tabular}

Fuente: Elaboración propia a partir de encuestas. 
Además, de acuerdo a los encuestados, los principales sobrecostos logísticos en el traslado se evidencian principalmente porque existen choques a la salida del puerto, por los excesos de velocidad y por equivocaciones de las rutas, lo que ocasiona que se desembolse montos adicionales de dinero que la empresa debe asumir ya que deben ingresar al taller a los vehículos siniestrados para su reparación con lo cual se incrementa el tiempo del despacho, dependiendo del tipo de daño, para posteriormente retirar la unidad vehicular.

De acuerdo a la tabla 5, se evidencia que el coeficiente de correlación de Spearman el cual se utilizó, muestra un valor de 0,153 el cual corrobora que existe una correlación positiva muy baja entre las variables "sobrecostos logísticos del terminal portuario" y el "tiempo de demora del despacho aduanero" de importación de los vehículos nuevos de la empresa Toyota, además, de mostrarnos una significancia bilateral de 0,379 ya que se aplicó a una población de 35 sujetos.

Entonces según lo mostrado por la tabla 5, la correlación existente entre las variables anteriormente mencionadas es positiva, es decir, que si una de ellas se incrementa también incrementará la otra variable y si por el contrario la primera disminuye, la segunda también lo hará.

De acuerdo a lo mostrado por la figura 3, se puede observar que el $94 \%$ del total de encuestados se encuentra de acuerdo con que los sobrecostos logísticos del terminal portuario se relacionan con el tiempo de demora del despacho aduanero, mientras que solo 1 persona representando el 3\% se mostró indiferente y la misma cantidad se mostró en desacuerdo. Vale la pena recalcar que los principales sobrecostos logísticos en el terminal son a causa de los choques dentro del terminal portuario, las abolladuras y los robos que se suscitan dentro del propio terminal.

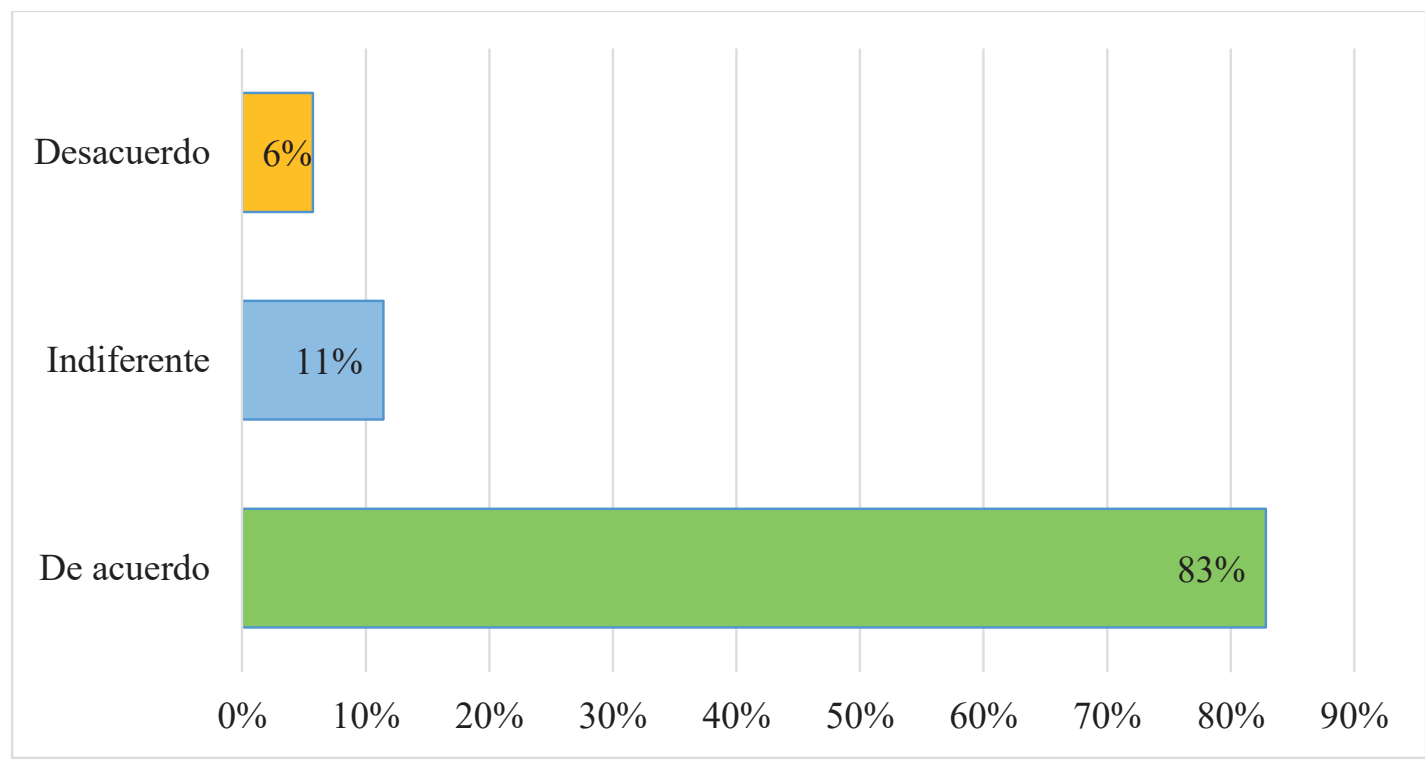

Figura 2. Los sobrecostos logísticos del traslado de los vehículos se correlacionan con el tiempo de demora del despacho aduanero

Fuente: Elaboración propia a partir de encuestas.

Tabla 5

Los sobrecostos logísticos del terminal portuario se correlacionan con el tiempo de despacho aduanero

\begin{tabular}{cll}
\hline \multicolumn{2}{l}{ Los sobrecostos logísticos del terminal portuario se correlaciona con el tiempo de despacho aduanero } & Tiempo de despacho aduanero \\
\hline & Coeficiente de correlación & 0,153 \\
Sobrecostos logísticos del terminal portuario & Sig. (bilateral) & 0,379 \\
& $\mathrm{~N}$ & 35 \\
\hline
\end{tabular}

Fuente: Elaboración propia en SPSS a partir de encuestas. 
Tabla 6

Los sobrecostos logísticos del terminal portuario se correlacionan con el tiempo de despacho aduanero

\begin{tabular}{|c|c|c|}
\hline RESPUESTAS & Frecuencia & Porcentaje \\
\hline De acuerdo & 33 & $94 \%$ \\
\hline Indiferente & 1 & $3 \%$ \\
\hline En desacuerdo & 1 & $3 \%$ \\
\hline TOTAL & 35 & $100.00 \%$ \\
\hline
\end{tabular}

Fuente: Elaboración propia a partir de encuestas.

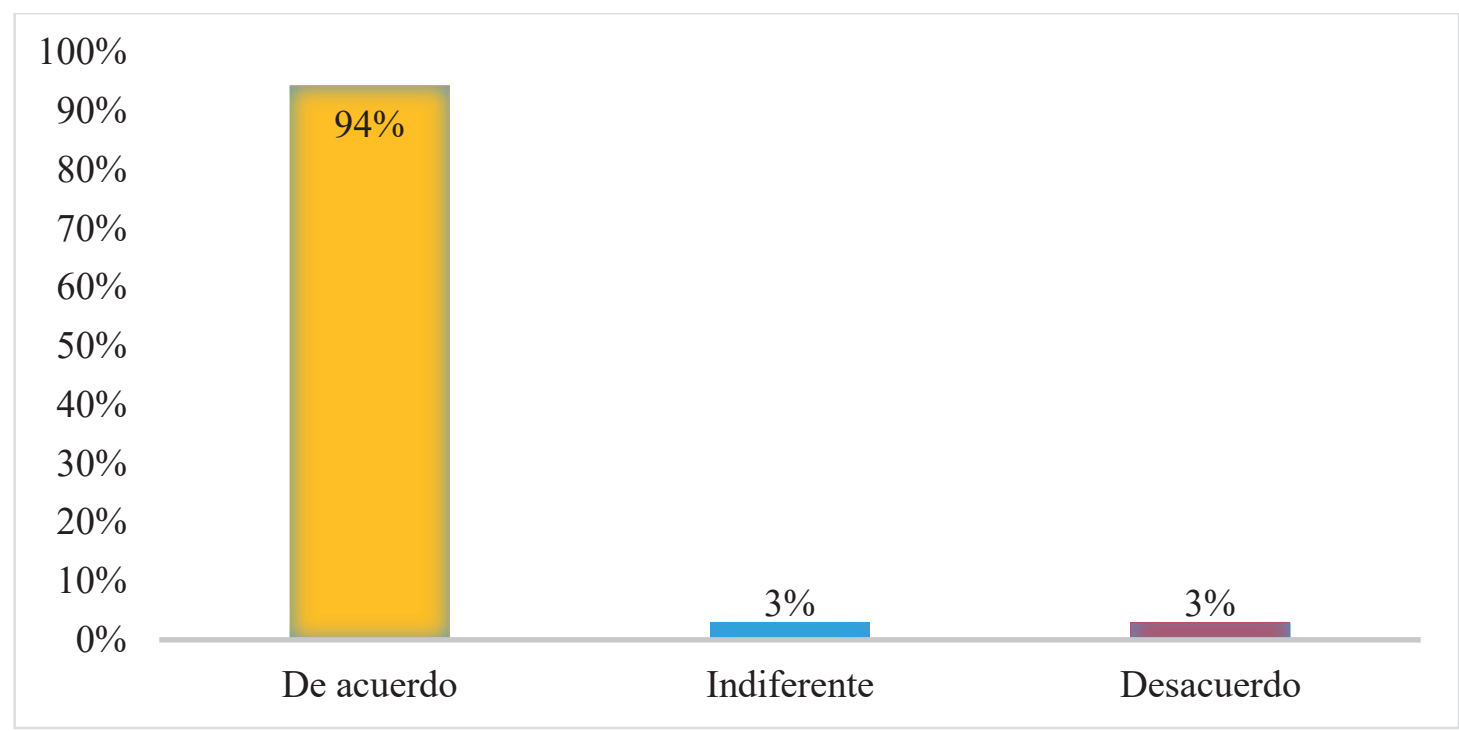

Figura 3. Los sobrecostos logísticos del terminal portuario se correlacionan con el tiempo de despacho aduanero

Fuente: Elaboración propia a partir de encuestas.

\section{DISCUSIÓN}

Los riesgos más resaltantes que se identificaron durante el despacho aduanero, se presentan usualmente en el área de operaciones externas, esto debido a que el personal del puerto quienes se encargan de conducir los vehículos desde que la nave tiene el permiso de descarga hasta el patio donde dejarán finalmente los vehículos para su posterior inspección, es donde se registran la mayor cantidad de siniestros, es decir, ese es el trayecto en el cual suceden choques o daños a las unidades por el mal manejo y/o descuido de parte de los estibadores al trasladar las unidades desde que llegan en el buque hasta el lugar en el patio del terminal en la cual se estacionarán hasta que se proceda con el retiro de las unidades vehiculares. De acuerdo a los resultados obtenidos, como lo menciona Núñez (2017), en nuestro país no se seguirían las recomendaciones brindadas por los principales organismos internacionales como son la Organización Mundial de Aduanas (OMA), la Comunidad Andina de Naciones (CAN) y la Organización para la Cooperación y el Desarrollo Económico (OCDE) cuyas recomendaciones se basan en la puesta en práctica de los procedimientos estandarizados que rigen a nivel internacional los cuales sirven para la facilitación del comercio exterior minimizando con ello los riesgos existentes en el procedimiento aduanero de mercancías. De acuerdo a lo mencionado por Huamán (2010) una empresa importadora se debe destacar por evitar los cuellos de botella para ser eficiente y con ello se economizan tiempos y costos para diferenciarse de su competencia, sin embargo, en los resultados de esta investigación, se identificó que una problemática constante en el despacho aduanero de vehículos es el servicio brindado por el terminal portuario en el área de operaciones externas, el cual genera demoras por falta de respuesta frente a los reclamos que realizan las empresas por daños o incidencias dentro del 
terminal o de envío de documentos de autorizaciones a tiempo. Una de las propuestas que se deben implementar en la empresa Toyota, es un benchmarking con los principales importadores de la competencia, ya que como lo referencia Boxwell, Rubiera, McShane y Zaratiegui (1995), con el benchmarking se pueden realizar no solo estudios comparativos con las áreas de las empresas de la competencia sino también para optimizar el funcionamiento en la propia organización.

\section{REFERENCIAS BIBLIOGRÁFICAS}

Álvarez, D. (2012). Organización del trabajo y dispositivos de control en el sector automotriz: el toyotismo como sistema complejo de racionalización. Trabajo y sociedad, (18), 43-57.

Barandiarán, M., Calderón, D., Chávez, G., \& Coello, A. (2013). Plan estratégico del sector automotriz en el Perú: vehículos ligeros y comerciales. (Tesis de Maestría). Pontificia Universidad Católica Del Perú, Lima.

Bloch, R. (2011). Las pequeñas y medianas empresas, la experiencia en Italia y en la Argentina. Buenos Aires: AD-HOC.

Boxwell, R., Rubiera, I., McShane, B., \& Zaratiegui, J. (1995). Benchmarking para competir con ventaja (No. 658.4/B78bE). New York: McGraw-Hill Education.

García, R. (2013). La mejora de la productividad en la pequeña y mediana empresa. Madrid: Editorial Club Universitario.

Hermosilla, R. (2015). El diseño del canal de distribución internacional como ventaja diferencial de marketing: caso Toyota. Gestión en el Tercer Milenio, 18(35), 65-72.
Hernández, S., Fernández, C., \& Baptista, M. (2014). Metodología de la Investigación (6ta ed.). México D.F.: McGraw-Hill Education.

Huamán, F. (2010). Estudio de pre factibilidad para industrializar y comercializar automóviles mediante una planta de ensamblaje automotriz en el Perú. (Tesis de pregrado). Pontificia Universidad Católica Del Perú, Lima.

Loaiza, M. (2007). Análisis, evaluación, y mejora de procesos logísticos de ingreso de mercadería bajo régimen de depósito autorizado en un operador logístico: teoría y ejemplo aplicativo. (Tesis de pregrado). Pontificia Universidad Católica Del Perú, Lima.

Nickl, M. (2005). La evolución del concepto "Logística" al de "Cadena de Suministros" y más allá. Compras y Existencias, 140(1).

Núñez, I. (2017). Impacto de la nueva regulación aduanera en el desaduanamiento en el régimen de importación de mercancías. Revista Espacios, 38(51), 14.

Pellegrini, F. (2011). El valor de las relaciones institucionales en un contexto de crisis organizacional. (Tesis de pregrado). Universidad del Aconcagua, Mendoza.

Quevedo, J. G. (2010). Análisis, diagnóstico y propuesta de mejora de la cadena logística y de planeamiento de las compras de una empresa peruana comercializadora de productos químicos. (Tesis de pregrado). Pontificia Universidad Católica Del Perú, Lima.

Rodríguez, C. (2013). Oligopolio y competencia mundial en la industria automotriz. La emergencia del Toyotismo y la caída del Fordismo. Economía Informa, 2013(383), 107-130. 
